\title{
Usefulness of Real Time PCR in diagnosing initial Pseudomonas aeruginosa infection in Cystic Fibrosis pediatric patients
}

\author{
Lucia Bassani', Domenico Colombrita', Emanuele Draghin', Laura Gazzola², \\ Arnaldo Caruso ${ }^{3}$, Rita Padoan ${ }^{2}$ \\ I. Laboratorio di Microbiologia Pediatrica, Ospedale dei Bambini, AO Spedali Civili, Brescia \\ 2. Centro di Supporto per la Fibrosi Cistica, Ospedale dei bambini, AO Spedali Civili, Brescia \\ 3. Struttura di Microbiologia, Dipartimento di Medicina Sperimentale ed Applicata, Università di Brescia
}

Key words: Cystic fibrosis, Real Time PCR, Pseudomonas aeruginosa, Initial infection, Eradication therapy

Utilità di Real Time PCR nel diagnosticare la prima infezione da Pseudomonas aeruginosa in pazienti pediatrici affetti da Fibrosi Cistica

\section{SUMMARY}

Chronic infection with Pseudomonas aeruginosa is the main agent responsible for lung function decline in Cystic Fibrosis patients. To prevent chronic colonization, timely identification of $P$. aeruginosa in the airways is crucial subsequently followed by eradication therapy. It is therefore necessary to use highly sensitive and specific methods. Our aim was to test the real-time PCR assay method to identify P. aeruginosa growth from CF respiratory specimens and compare it with conventional microbiological cultures.

754 sputum samples from 96 CF paediatric patients were processed between November 2009 and April 20 I I with Real-Time PCR assays in addition to conventional microbiological cultures. Patients were categorized according to their P. aeruginosa infection status in three groups: "chronic infection", "intermittent infection", "negative".

From 16 chronic patients, seven cultures out of 138 (5\%) were found to be positive using RT-PCR and then negative after cultures; from 29 intermittent patients, 18 out of 59 (30.5\%) samples were positive only using the PCR method; from 5 I negative patients, I 3 out of 39 (33\%) specimens were positive only with PCR. No false negative PCR results were detected when conventional cultures were positive.

Real-Time PCR test was more sensitive in identifying P. aeruginosa in "intermittent infection" or "negative" patients. Clinical trials are needed to explore the usefulness of starting eradication Pseudomonas therapy on the basis of only a positive PCR test, without waiting for the bacterial growth on conventional microbiological cultures. The usefulness of real time PCR could also be tested in the follow-up of eradication therapy efficacy.

\section{INTRODUCTION}

Cystic Fibrosis (CF) is an autosomic recessive disease in Caucasian individuals, which arises from mutations in the cystic fibrosis trans-membrane conductance regulator gene (CFTR) located on chromosome 7 (17). It encodes for a protein functioning principally as a chloride channel. The absence or malfunction of the CFTR protein leads to abnormally viscous mucus by the affected glands, mainly causing chronic lung disease and pancreatic insufficiency (16).

The principal clinical complication is the chronic lung disease due to recurrent bacterial infections. Chronic endobronchial infection by bacteria, viruses, fungi and chronic inflammation are responsible for progressive structural damage of the lung structure and progressive loss of respiratory function, leading to increased morbidity, res- piratory failure and mortality of CF patients (14). During the first ten years of CF patients, Staphylococcus aureus and Haemophilus influenzae are the most common bacteria isolated from the sputum, but in the second and third decade of life, $P$. aeruginosa is the prevalent bacteria among Gram negative and/or opportunistic pathogens such as Stenotrophomonas maltophilia or Achromobacter xylosoxidans. A distinct role has Burkholderia cepacia, which may lead to severe acute or chronic lung disease. A secondary role may be played by respiratory syncytial virus, influenza virus, atypical mycobacteria, fungi, such as Aspergillus spp $(4,9)$.

Chronic infection with $P$. aeruginosa is the main agent responsible for lung function decline and ultimate mortality in CF patients (19). The first infection is usually started by non-mucoid "rough"

\section{Corresponding author: Rita Padoan}

Cystic Fibrosis Support Centre, Children's Hospital, AO Spedali Civili - Brescia,

Piazzale Spedali Civili I, 25I 23 Brescia, Italy - Tel.: +390303996287 - Fax: +390303996028

E-mail: ritaf54@gmail.com 
strains, which are motile and prototrophic and usually sensitive to a variety of anti-microbials (2). Chronic colonization by mucoid $P$. aeruginosa growing as bio-films in the airways of CF patients (10) is very difficult to eradicate $(14,18)$.

The time to identify $P$. aeruginosa in CF airways is crucial in order to prevent its chronic colonization achieved by starting the eradication therapy early (11) and so we need to use highly sensitive and specific methods. Some authors suggest using molecular methods to aid in the identification of bacterial isolates from microbiologic cultures (3, 15, 20). Real-time polymerase chain reaction (PCR) assays have shown their potential to detect $P$. aeruginosa earlier than culture methods $(1,5$, 13). Otherwise, others suggest that using PCR to identify $P$. aeruginosa directly from clinical specimens gives no advantage over conventional methods, i.e., biochemical identification from microbiologic culture (6).

To clarify the usefulness of Real-Time PCR assay methodology, in identifying $P$. aeruginosa in bronchial specimens from CF paediatric patients, we tested and compared it with conventional microbiological cultures.

\section{METHODS}

\section{Patients}

Bronchial secretions were obtained by naso-pharyngeal aspiration in non-expectorating children or sputum samples from $96 \mathrm{CF}$ patients.

Samples were collected every two months in children and every three months in adults (21), as part of their routine clinical care at our Children's Hospital. Patients were categorized in three groups according to their $P$. aeruginosa infection status in the previous year: "chronic infection", with 3 or more positive samples; "intermittent infection" with less than $50 \%$ of positive samples; "negative" samples with no $P$. aeruginosa positive cultures (free of $P$. aeruginosa infection or never infected) (12).

\section{Microbiological methods}

Overall, 754 samples from $96 \mathrm{CF}$ patients were processed between November 2009 and April 2011 with Real-Time PCR assay compared with conventional microbiological cultures.

Specimens were tested to detect bacterial pathogens using conventional laboratory methods and tested in Real-Time PCR only to detect $P$. aeruginosa. Sputum samples were first homogenated in the homogenizer Medifasth (Consul), and then all samples were treated as explained. Five hundred microliters were inoculated into a lysis buffer (bioMérieux) to lisate bacteria before DNA extraction with Nuclisens EasyQ, Minimag semi-automatic extraction
(bioMérieux) and 20 microliters were inoculated in each selective and non-selective media to isolate $P$. aeruginosa. All plates were incubated at $37^{\circ} \mathrm{C}$ in $5 \% \mathrm{CO}_{2}$ for 72 hours and assessed for clinically relevant organisms identified with GNI biochemical tests (ATB bioMérieux).

The DNA samples extracted were analyzed for $P$. aeruginosa by a qualitative Real-Time PCR assay, BioDetect $P$. aeruginosa (Biodiversity), according to the manufacturer's instructions on the 7500 Real-Time PCR System instrument (Applied Biosystem). This kit has been designed in a conserved region of the gene oprL, coding for a protein of the outer membrane. The assay is based on DNA amplification and fluorescent detection of products in a single tube amplification.

The assay for $P$. aerginosa has a positive control of amplification with a defined concentration of specific molecular targets. A possible inhibition of DNA extraction and amplification can be detected by using an internal control. Each sample was tested in Real-Time in duplicate, as were negative control samples already taken from DNA extraction.

\section{RESULTS}

All 754 samples were collected in 18 months from $96 \mathrm{CF}$ patients, aged 5-25 years. Of these samples, 159 samples $(21 \%)$ were from 16 chronic patients with a mean age of 17.7 years; 191 samples $(25 \%)$ were from 29 intermittent patients with a mean age of 8 years; 404 samples (54\%) were from 51 negative children with a mean age of 6 years.

One hundred and thirty-one samples from the chronic patient group were positive from the PCR and cultures, 21 were negative with the PCR and cultures, but only 7 of $138(5 \%)$ were positive with the PCR and negative cultures.

Of the 191 samples from the 19 intermittent patients, 41 were positive using both methods, 132 were negative with both methods, but 18 of $59(30.5 \%)$ were positive using only the PCR method. Of 404 samples from the 51 negative patients, 26 were positive with both methods, 365 were negative with both methods, and 13 of 39 (33\%) were positive only with the PCR method (Table 1).

No false negative PCR results were detected when the conventional culture was positive but in total 38 specimens were PCR positive with a negative culture growth. We improved identification of $P$. aeruginosa by $5 \%, 44 \%$ and $50 \%$, in the three patients' groups respectively.

\section{DISCUSSION}

In this paper we have shown the usefulness of Real-Time PCR test to identify $P$. aeruginosa DNA, in patients categorized as "intermittent 
infection" or "free of $P$. aeruginosa". In both these groups, there was a positivity increase of $44 \%$ and $50 \%$ respectively.

The altered function of the chloride channel in $\mathrm{CF}$ airways diminished chloride and water secretion leading to viscous secretions and impaired mucociliary clearance $(14,16,19)$.

Therefore, from early childhood, CF patients are prone to recurrent and chronic respiratory tract infections. Infections caused by $P$. aeruginosa may persist and have been correlated to a lower clinical score, lower predicted $\mathrm{FEV}_{1}$ percent contributing to morbidity and leading to respiratory failure and ultimately death.

As a result, a great effort has made in all CF Centers to prevent and treat it. When treated precociously, there is a higher possibility to eradicate it and thus postpone chronic colonization.

Early antibiotic treatment at the first isolation of the germ (eradication therapy) reduces it becoming chronic $(8,11)$. At first colonization, eradication is more frequent due to its lower bacterial charge and "non-mucoid" phenotype which is more susceptible to antibiotic treatment.

In order to face the $P$. aeruginosa challenge properly a close cooperation between clinicians and microbiologists is essential, to find the best method of early and accurate diagnosis (7).

In our centre, about 100 patients are regularly seen, they all receive a sputum culture at each clinical visit (at least quarterly).

In order to reduce the risk of cross-infection, patients are categorized into chronic infection, intermittent, and negative groups for $P$. aeruginosa isolation.

In order to test Real-Time PCR assay methodology, in order to identify $P$. aeruginosa strains in $\mathrm{CF}$ children earlier, we compared it with conventional microbiological cultures. We found a greater sensitivity of Real-Time PCR to cultures in identifying also a very low bacterial charge; moreover it allows an earlier diagnosis. Particularly in our 7 subjects, classified as negative, RT PCR detected the $P$. aeruginosa presence before the conventional microbiological test did. When these data are confirmed, RT PCR results could also be used as a timely start of eradication therapy.

Our results also showed that the PCR method is not useful in CF patients with chronic infection (only 5\% PCR positive with a negative culture), while it would be better used in CF patients with an intermittent infection (44\% of increase in identification) or in CF patients categorized as "negative" (50\% of increase).

Although the conventional microbiological culture is still the gold standard diagnostic test for bacterial endobronchial infection in $\mathrm{CF}$ patients, the RT-PCR diagnostic test has $100 \%$ specificity and more than $90 \%$ sensitivity.

The assay needs to treat the sputum sample appropriately to homogenise it and also to use a valid extraction method, otherwise it may be not equally precise as the microbiological culture.

Due to the importance of proper $P$. aeruginosa diagnosis, the PCR assay could be used to control a negative culture.

In conclusion, the use of Real-Time PCR increases the detection rate of $P$. aeruginosa from samples collected from $\mathrm{CF}$ patients without chronic colonization.

Our results also showed that much more attention needs to be paid to segregation measures for not colonized patients, in order to reduce the risk of cross-infection.

Further randomized clinical trials are needed to explore the usefulness of starting eradication $P$. aeruginosa. Therapy using only PCR positive tests. Subsequently it should be clarify if this approach is able to delay or prevent chronic colonization by $P$. aeruginosa. The usefulness of RealTime PCR could also be tested in the follow-up of eradication therapy efficacy.

Table I. Results of airway secretions samples tested for P. aeruginosa in the three patients' group.

\begin{tabular}{lllllll}
\hline & Chronic (159 samples) & Intermittent (I9I samples) & Free (404 samples) \\
\hline & PCR & \multicolumn{1}{l}{ PCR } & & PCR \\
\hline culture & + & - & + & - & + & - \\
\hline+ & I3I & 0 & 41 & 0 & 26 & 0 \\
\hline- & 7 & 21 & 18 & 132 & 13 & 365 \\
\hline
\end{tabular}

\section{Contributors' statement:}

RP has the clinical responsibility of Cystic Fibrosis patients, discussed clinical and microbiological data, revised critically the draft

LG participated in clinical follow up of patients, collected data and contributed in bibliographic research

LB and DC devised the study project, wrote and discussed the draft

LB performed PCR test, performed bibliographic research,

DC, ED performed microbiological investigation,

AC contributed in the project development 


\section{Acknowledgements}

LB has a grant from Associazione Lombarda Fibrosi Cistica - onlus to perform real-time PCR for $P$. aeruginosa.

We would like to thank Dott. Robert Coates for his linguistic revision of the paper.

\section{REFERENCES}

1. Billard-Pomares T, Herwegh S, Wizla-Derambure N, Turck D, Courcol R, Husson MO. Application of quantitative PCR to the diagnosis and monitoring of Pseudomonas aeruginosa colonization in 5-18-yearold cystic fibrosis patients. J Med Microb 2011; 60: 157-61.

2. Burns JL, Gibson RL, McNamara S, et al. Longitudinal assessment of $P$. aeruginosa in young children with cystic fibrosis. J Infect Dis 2002; 183: 444-52.

3. Clarke L, Moore JE, Millar BC, et al. Development of a diagnostic PCR assay that targets a heat-shock protein gene (groES) for detection of Pseudomonas spp in cystic fibrosis patients. J Med Microbiol 2003; 52: 759-63.

4. Cystic Fibrosis Patients Registry 2010 Annual Data Report Bethesda Maryland 2011 Cystic Fibrosis Foundation.

5. da Silva Filho LV, Tateno AF, et al. The combination of PCR and serology increases the diagnosis of $P$. aeruginosa colonization/infection in cystic fibrosis. Pediatr Pulmonol 2007; 42: 938-44.

6. Deschagt $\mathrm{P}$, De Baere T, Van Simaey L, et al. Comparison of the sensitivity of culture, PCR and quantitative Real-Time PCR for the detection of $P$. aeruginosa in sputum of cystic fibrosis patients. $B M C$ Microbiol 2009; 9: 244-50.

7. Deschaght P, Van Daele S, De Baets F, Vaneechoutte M. PCR and the detection of Pseudomonas aeruginosa in respiratory samples of CF patients. A literature review. J Cyst Fibr 2011; 10: 293-7.

8. Frederiksen B, Koch C, Hoiby N. Antibiotic treatment of initial colonization with Pseudomonas aeruginosa postpones chronic infection and prevents deteriorament of pulmonary function in cystic fibrosis. Pediatr Pulmonol 1997; 23: 330-5.
9. Gilligan P.H. Microbiology of airway disease in patients with cystic fibrosis. Clin Microbiol Rev 1991; 4: 35-51.

10. Høiby N. Recent advances in the treatment of Pseudomonas aeruginosa infections in cystic fibrosis. BMC Med. 2011; 9: 32.

11. Koch C, Høiby N. Prevention of chronic Pseudomonas aeruginosa colonisation in cystic fibrosis by early treatment. Lancet 1991; 338: 725-6.

12. Lee TWR, Brownlee KG, Conway SP, Denton M, Littlewood JM. Evaluation of a new definition for chronic Pseudomonas aeruginosa infection in cystic fibrosis patients. J Cyst Fibr 2003; 2: 29-34.

13. McCulloch E, Lucas C, Ramage G, Williams C. Improved early diagnosis of Pseudomonas aeruginosa by Real-Time PCR to prevent chronic colonisation in a paediatric cystic fibrosis population. J Cyst Fibr 2011; 10: 21-4.

14. O'Sullivan BP, Freedman SD. Cystic fibrosis. Lancet 2009; 373: 1891-904.

15. Qin X, Emerson J, Stapp J, Stapp L, Abe P, Burns JL. Use of Real-Time PCR with multiple targets to identify $P$. aeruginosa and other nonfermenting Gram-negative bacilli from patients with cystic fibrosis. J Clin Microbiol 2003; 41: 4312-7.

16. Ratjen F, Doring G. Cystic Fibrosis. Lancet 2003; 361: 681-9.

17. Rommens JM, Zengerling S, Burns J, et al. Identification and regional localization of DNA markers on chromosome 7 for the cloning of the cystic fibrosis gene. Am J Hum Genet 1988; 43: 645-63.

18. Rybtke MT, Jensen PØ, Høiby N, Givskov M, TolkerNielsen T, Bjarnsholt T. The implication of Pseudomonas aeruginosa biofilms in infections. Inflamm Allergy Drug Targets 2011; 10: 141-57.

19. Sheppard MN. The pathology of cystic fibrosis. In: Hodson ME, Geddes D, eds. Cystic Fibrosis. London, Chapman and Hall; 1995: 131-49.

20. Spilker T, Coenye T, Vandamme P, Lipuma JJ. PCRbased assay for differentiation of Pseudomonas aeruginosa from other Pseudomonas species recovered from cystic fibrosis patients. J Clin Microbiol 2004; 42: 2074-9.

21. The CF Trust's Clinical Standards and Accreditation Group. Standards of care: standards for the clinical care of children and adults with $\mathrm{CF}$ in the UK. CF Trust, London UK, 2001. 